\section{UK retinopathy of prematurity guideline}

For more information and to view the full document, please see http://www.rcophth.ac.uk/about/publications

\section{Executive summary}

Retinopathy of prematurity (ROP) is one of the few causes of childhood visual disability which is largely preventable. Many extremely preterm babies will develop some degree of ROP although in the majority this never progresses beyond mild disease which resolves spontaneously without treatment. A small proportion develop potentially severe ROP which can be detected through retinal screening. If untreated, severe disease can result in serious vision impairment and consequently all babies at risk of sight-threatening ROP should be screened.

This evidence-based guideline for the screening and treatment of ROP was developed by a multidisciplinary guideline development group (GDG) of the Royal College of Paediatrics \& Child Health (RCPCH) in collaboration with the Royal College of Ophthalmologists (RCOphth), British Association of Perinatal Medicine (BAPM) and the premature baby charity BLISS. The guideline was produced according to RCPCH standards for guideline development. ${ }^{1}$

The guideline provides 25 evidence-based recommendations and 21 good practice points. Recommendations are graded A-D using SIGN grading hierarchy, ${ }^{2}$ according to the strength of the evidence underpinning them. The good practice points (GPP) are a consensus of the GDG. This Executive Summary highlights those recommendations and good practice points considered by the GDG to be priorities for implementation.

This guideline has been produced specifically for use within the UK and supersedes the previous guideline. ${ }^{3}$ It will not be applicable in countries where more mature babies are at risk of sight threatening ROP. ${ }^{4}$

Not all the recommendations are included in this Summary. The full Guideline should be consulted which also contains complete details of the Guideline methodology. Appendices A, B, C and D give a standardised sheet for recording screening results, an algorithm for ophthalmic criteria for screening and treatment, the International Classification of ROP Revisited, ${ }^{5}$ and parent information leaflets respectively. All the documents are available on the websites of the Royal College of Ophthalmologists www.rcophth.ac.uk, the Royal College of Paediatrics and Child Health www.rcpch.ac.uk or the British Association of Perinatal Medicine www.bapm.org.

\section{Key recommendations/good practice points for implementation}

\subsection{Screening criteria}

- All babies less than 32 weeks gestational age (up to 31 weeks and 6 days) or less than $1501 \mathrm{~g}$ birthweight should be screened for ROP. One criterion to be met for inclusion.

GPP

- All babies less than 31 weeks gestational age (up to 30 weeks and 6 days) or less than $1251 \mathrm{~g}$ birthweight must be screened for ROP. One criterion to be met for inclusion.

\subsection{Screening protocol}

- Babies born before 27 weeks gestational age (i.e. up to 26 weeks and 6 days) - the first ROP screening examination should be undertaken at 30 to 31 weeks postmenstrual age.

- Babies born between 27 and 32 weeks gestational age (i.e. up to 31 weeks and 6 days) - the first ROP screening examination should be undertaken between 4 to 5 weeks (i.e. 28-35 days) postnatal age.

- Babies > 32 weeks gestational age but with birthweight $<1501$ grams - the first ROP screening examination should be undertaken between 4 to 5 weeks (i.e. 28-35 days) postnatal age.

- Minimum frequencies of screening should be weekly when:

- the vessels end in zone I or posterior zone II; or

- there is any plus or pre-plus disease or

- there is any stage 3 disease in any zone

- Minimum frequencies of screening should be every 2 weeks:

- In all other circumstances until the criteria for termination have been reached

- All babies $<32$ weeks gestational age or birthweight $<1501 \mathrm{~g}$ should have their first ROP screening examination prior to discharge.

Although screening for all babies at risk should follow the above protocol, it is acknowledged that there may be clinical or organisational circumstances which prevent this. In these circumstances the following is recommended as good practice to ensure that subsequent screening examinations are not missed.

- Where a decision is made not to screen a baby, the reasons for doing so should be clearly stated in the baby's medical record and the examination should be rescheduled within one week of the intended examination.

GPP

\subsection{Screening examination}

The screening examination can be stressful for both babies and parents. The full guideline gives recommendations on preparation and care of the baby. The examination requires a well-dilated pupil so the peripheral retina can be fully visualised. The following are key recommendations and good practice points for this area.

- In addition to oral communication, parents should be given written information about the screening process prior to the first examination of their baby. GPP

- It is important that the periphery of the retina can be seen and this may be facilitated by the use of an eyelid speculum and scleral indentor suitable for neonatal use.

- Ophthalmological notes should be made after each ROP examination, detailing zone, stage, and extent in terms of clock hours of any ROP and the presence of any pre-plus or plus disease. These notes should include a recommendation for the timing of the next 
examination (if any) and be kept with the baby's medical record.

GPP

- Comfort care techniques (e.g. administering sucrose solution, nesting, swaddling and/or the use of a pacifier) during the screening examination may be considered.

\subsection{Termination of ROP screening}

Screening can be stopped when a baby is no longer at risk of sight-threatening ROP.

In babies who never develop any ROP, the risk of sight-threatening ROP developing is minimal once the retinal vessels have entered zone III. That vessels are in zone III can be difficult to determine, but it is unlikely to occur before 37 weeks postmenstrual age and a decision to stop screening before this must be carefully evaluated.

- In babies without ROP, there is minimal risk of developing sight-threatening ROP when vascularisation has extended into zone III and eye examinations may be stopped when this happens, usually after 36 completed weeks postmenstrual age.

In babies developing ROP which does not meet the criteria for treatment, screening can be safely stopped when there are clear signs that the active progression of ROP has halted and regression has commenced.

- In the presence of ROP, screening for progressive active disease may be discontinued when any of the following characteristics of regression are seen on at least 2 successive examinations:

- lack of increase in severity

- partial resolution progressing towards complete resolution

- change in colour in the ridge from salmon pink to white

- transgression of vessels through the demarcation line

- commencement of the process of replacement of active ROP lesions by scar tissue

\subsection{ROP treatment}

Timely treatment for ROP is effective at preventing severe vision impairment. Previous guidance recommended treatment when the disease reached 'Threshold', as defined in section 7 of the main document. ${ }^{6}$ Recent evidence shows benefit from earlier treatment.

\subsection{Ophthalmic criteria for treatment}

- Treatment for ROP should be undertaken if any of the following indications are reached:

- Zone I, any ROP with plus disease,

- Zone I, stage 3 without plus disease,

- Zone II, stage 3 with plus disease.

- Treatment for ROP should be seriously considered if the following indication is reached:

- Zone II, stage 2 with plus disease

Although there is no specific evidence to inform the interval between reaching treatment criteria and treatment taking place, it is the view of the GDG that, given the encouraging results for early treatment obtained by treating within 48 hours, this should be the target standard.

- Babies with aggressive ROP (as defined in ICROP revisited) should be treated as soon as possible and within $48 \mathrm{~h}$. ROP requiring treatment but which is not aggressive posterior ROP should normally be treated within $48-72 \mathrm{~h}$.

GPP

- Transpupillary diode laser therapy is recommended as the first line treatment for ROP.

- Treatment with near-confluent (0.5-1 burn-width) laser burn spacing should be administered to the entire avascular retina.

- The unavailability of diode laser equipment or the inability to transfer to another centre should not prevent or delay the treatment of ROP. In these situations, treatment with cryotherapy or argon laser may be completed by an ophthalmologist experienced in these techniques.

GPP

Severe ROP requiring treatment is relatively infrequent and treatment is a specialised procedure. Although there is no research literature on treatment outcomes according to operator expertise, it is likely that those with the greatest experience will be the most skilled practitioners in the procedure.

- Babies with ROP should be treated by ophthalmologists who have the appropriate competency. GPP

- Each network should have identified individuals for ROP treatment

GPP

\subsection{Post treatment reviez}

Post operative review is important to monitor disease regression and to determine if retreatment is necessary. The GDG have agreed the following GPP in the absence of good quality evidence to inform these timings.

- The first examination post treatment should take place 5-7 days after treatment and should be continued at least weekly for signs of decreasing activity and regression.

GPP

- Re-treatment should be performed usually 10-14 days after initial treatment when there has been a failure of the ROP to regress.

GPP

\subsection{Follow-up after screening or treatment}

- After the acute phase, eyes that have reached stage 3 or have been treated should be monitored at a frequency dictated by the clinical condition to determine the risk of sequelae.

GPP

\subsection{Organisation of services}

Effective services for ROP screening and treatment must be embedded in a robust organisational structure, with individual responsibilities identified. Particular efforts must be made to ensure that the service is delivered appropriately for all those at risk, as there is evidence that babies transferred or discharged home before 
screening is complete are at risk of poor outcomes as a result of lack of follow-up.

- All units caring for babies at risk of ROP should have a written protocol in relation to the screening for, and treatment of, ROP. This should include responsibilities for follow-up of babies transferred or discharged from the unit before screening is complete, which should be the responsibility of the named consultant neonatologist for each baby.

GPP

- If babies are transferred either before ROP screening is initiated or when it has been started but not completed, it is the responsibility of the consultant neonatologist to ensure that the neonatal team in the receiving unit is aware of the need to start or continue ROP screening.

GPP

- There should be a record of all babies who require review and the arrangements for their follow-up.GPP

- For babies who meet the ROP screening criteria, screening status and the need and arrangements for further screens must be recorded in all transfer letters so that screening may be continued.

- For babies discharged home before screening is complete the first follow-up out-patient appointment must be made before hospital discharge and the importance of attendance explained to the parents/carers.

\subsection{Work commitment}

- Ophthalmologists regularly completing ROP screening and/or treatment should have sessional commitments allocated within their work plan.

GPP

On behalf of a Guideline Development Group of the Royal College of Paediatrics and Child Health, the Royal College of Ophthalmologists and the British Association of Perinatal Medicine.

\section{References}

1 Royal College of Paediatrics and Child Health. Standards for development of Clinical guidelines in paediatrics and child health. RCPCH: London, June 2006.

2 Scottish Intercollegiate Guidelines Network. Sign 50: A guideline developers' Handbook. SIGN: Edinburgh, 2001.
3 The report of a Joint Working Party of The Royal College of Ophthalmologists and the British Association of Perinatal Medicine. Retinopathy of prematurity: Guidelines for screening and treatment. Early Hum Dev 1996; 46(3): 239-258.

4 Gilbert C, Fielder A, Gordillo L, Quinn G, Semiglia R, Visintin $\mathrm{P}$ et al. Characteristics of infants with severe retinopathy of prematurity in countries with low, moderate and high levels of development: implications for screening programs. Pediatrics 2005; 115(5): e518-e525.

5 International Committee for the Classification of Retinopathy of Prematurity. The International Classification of Retinopathy of Prematurity revisited. Arch Ophthalmol 2005; 123(7): 991-999.

6 Cryotherapy for Retinopathy of Prematurity Cooperative Group. Multicenter trial of cryotherapy for retinopathy of prematurity: Preliminary results. Arch Ophthalmol 1988; 106(4): 471-479.

AR Wilkinson'1 L L Haines², K Head² and AR Fielder ${ }^{3}$

${ }^{1}$ Department of Paediatrics, University of Oxford, Neonatal Unit, John Radcliffe Hospital, Oxford, UK ${ }^{2}$ Research Unit, Royal College of Paediatrics \& Child Health, London, UK

${ }^{3}$ Department of Ophthalmology, City University, St Mary's and Hillingdon Hospitals, London, UK

Correspondence: Professor AR Wilkinson, Professor of Paediatrics and Perinatal Medicine, University of Oxford, Neonatal Unit, Women's Centre, John Radcliffe Hospital, Oxford OX3 9DU, UK.

E-mail: andrew.wilkinson@paediatrics.ox.ac.uk

Reprinted from Early Human Development 84, AR Wilkinson, L Haines, K Head, AR Fielder, UK retinopathy of prematurity guideline, 71-74, Copyright (2008), with permission from Elsevier.

Eye (2009) 23, 2137-2139; doi:10.1038/eye.2008.128; published online 3 October 2008 